\author{
Effect of Ion-Beam Parameters on In-Plane Texture of \\ Yttria-Stabilized Zirconia Thin Films* \\ T. G. Truchan, M. P. Chudzik, B. L. Fisher, R. A. Erck, \\ K. C. Goretta, and U. Balachandran \\ Energy Technology Division \\ Argonne National Laboratory, Argonne, IL, USA
}

September 2000

The submitted manuscript has been created by the
University of Chicago as Operator of Argonne National
Laboratory ("Argonne") under Contract No. W-31-109-
ENG-38 with the U.S. Department of Energy. The U.S.
Government retains for itself, and others acting on its
behalf, a paid-up, nonexclusive, irrevocable worldwide
license in said article to reproduce, prepare derivative
works, distribute copies to the public, and perform publicly
and display publicly, by or on behalf of the Government.

Paper to be presented at Applied Superconductivity Conference ASC 2000, Virginia Beach, VA, September 17-22, 2000; to be published in IEEE Trans. Appl. Supercond.

*Work supported by the U.S. Department of Energy (DOE), Energy Efficiency and Renewable Energy, as part of a DOE program to develop electric power technology, and the Argonne Division of Educational Programs with funding from DOE, under Contract W-31-109-Eng-38. 


\section{DISCLAIMER}

This report was prepared as an account of work sponsored by an agency of the United States Government. Neither the United States Government nor any agency thereof, nor any of their employees, make any warranty, express or implied, or assumes any legal liability or responsibility for the accuracy, completeness, or usefulness of any information, apparatus, product, or process disclosed, or represents that its use would not infringe privately owned rights. Reference herein to any specific commercial product, process, or service by trade name, trademark, manufacturer, or otherwise does not necessarily constitute or imply its endorsement, recommendation, or favoring by the United States Government or any agency thereof. The views and opinions of authors expressed herein do not necessarily state or reflect those of the United States Government or any agency thereof. 


\section{DISCLAIMER}

Portions of this document may be illegible in electronic image products. Images are produced from the best available original document. 


\title{
Effect of Ion-Beam Parameters on In-Plas TI Texture of Yttria-Stabilized Zirconia Thin Films
}

\author{
T. G. Truchan, M. P. Chudzik, B. L. Fisher, R A. Erck, K. C. Goretta, and U. Balachandran
}

\begin{abstract}
Biaxially textured thin films of 8-mole $\%$-yttriastabilized zirconia (YSZ) were deposited by ion-beam-assisted deposition (IBAD) on polished Hastelloy-C tapes. These film s serve as epitaxial template layers for highly textured $\mathrm{Y}-\mathrm{Ba}-\mathrm{Cu}-$ $O$ superconductor thin films. YSZ films were deposited to a gross thickness of $\approx 1.6 \mu \mathrm{m}$ by electron beam evaporation. $A$ $300-e V$ Ar $110 \% \quad \mathrm{O}_{2}$ ion beam bombarded the substrate at an off-normal angle during deposition. The ion-to-atom arrival ratio ( $r$-value) was varied by independently adjusting the deposition rate and the ion current density. X-ray pole figures and $\phi$ scans were used to investigate in-plane texture. Profilometry and spectral reflectivity were utilized to measure the net film thickness. A two-dimensional texture/thickness contour map was generated and used to optimize the in-plane texture of the YSZ and to minimize processing time.
\end{abstract}

Index Terms-Biaxial Texture, IBAD, Coated Conductors

\section{INTRODUCTION}

C OATED conductors that are fabricated by thin-film techniques provide a route to successful development of superconductor wires [1]. Coated conductors emerged during attempts to develop the biaxial texture in $\mathrm{YBa}_{2} \mathrm{Cu}_{3} \mathrm{O}_{\mathrm{x}}$ (YBCO) that is necessary for optimal current flow. The processing involves depositing thick $\mathrm{YBCO}$ films onto a well-textured template, resulting in well-textured YBCO. The alignment and electrical performance of the YBCO depends on the texture of the template layer [2]. Buffer layers are necessary to relieve the geometrical mismatches between the metallic substrate and the YBCO layer, and provide the template for epitaxial YBCO deposition. Through proper

Manuscript submitted September 18,2000. Work supported by the U.S. Department of Energy (DOE), Energy Efficiency and Renewable Energy, as part of a DOE program to develop electric power technology, and the Argonne Division of Educational Programs with funding from DOE, under Contract W-31-109-Eng-38.

T. G. Truchan was with Argonne National Laboratory, Argonne, IL 60439, USA. He is now with Opto Power Corporation, 3321 E. Global Loop. Tucson AZ 85706, USA (telephone: 520-746-1234; email: info-opc@optopower.com).

M. P. Chudzik was with Argonne National Laboratory, Argonne, IL 60439, USA. He is now with IBM Corporation, East Fishkill, NY 12533, USA (telephone: 845-892-5000; e-mail: michaelchudzik@hotmail.com).

B. L. Fisher is with the Energy Technology Division, Argonne National Laboratory, Argonne, IL 60439, USA (telephone: 630-252-5029; e-mail: bfisher@anl.gov).

R. A. Erck is with the Energy Technology Division, Argonne National Laboratory, Argonne, IL 60439, USA (telephone: 630-252-4972; e-mail: erck@anl.gov).

K. C. Goretta is with the Energy Technology Division, Argonne National Laboratory, Argonne, IL 60439, USA (telephone: 630-252-7761; e-mail: goretta@anl.gov).

U. Balachandran is with the Energy Technology Division, Argonne National Laboratory, Argonne, IL 60439, USA (telephone: 630-252-4250; e-mail: balu@anl.gov). control of processing conditions, deposition on buffer layers of (100) orientation normal to a surface with adequate lattice match yields properly oriented YBCO thin films [2].

The lattice parameter of 8-mol\%-yttria-stabilized zirconia (YSZ) is $5.14 \AA$. Matching of (100) surfaces dictates a $45^{\circ}$ rotation of YBCO, and, with a lattice parameter of $\sqrt{2} \mathrm{a}_{0}=$ $5.4 \AA$, the degree of mismatch is sufficiently low to make YSZ a viable buffer layer material.

Ion-beam-assisted deposition (IBAD) is an established method for developing reproducible in-plane texture in YSZ long-length buffer layers [1]. Bombardment of the surface by energetic ions provides energy to the arriving adatoms through atomic collisions and allows them to coalesce, nucleate, and grow into a dense, hard film. Substrate heating is not necessary for film growth, which allows processing to be performed at room temperature.

IBAD relies on the off-normal bombardment of low-energy ions in conjunction with physical vapor deposition to induce a preferred texture in a deposited film [1,3]. Extensive investigations have been performed to extend this method to large substrates. This technique is used to produce highly oriented films of YSZ in which the [100] axis is aligned with the substrate normal, and the [111] axis with the bombarding beam axis. YBCO films that exhibit in-plane texture within $\approx 10^{\circ}$ full width at half maximum (FWHM) of perfect orientation can have critical current densities $>3 \times 10^{6} \mathrm{~A} / \mathrm{cm}^{2}$ and critical currents $>100 \mathrm{~A}$ at $77 \mathrm{~K}$ [1],[3].

Previous work has shown that the degree of texture in YSZ produced by IBAD depends primarily on the ratio of ions to atoms (r-value) arriving at the substrate surface during processing. The texture can be varied by changing the current density of the ion beam and the deposition rate. In-plane alignment of YSZ varies significantly with the incident angle of the ion beam, and shows a maximum preferred orientation at a bombardment angle of $55^{\circ}$, which corresponds to the channeling direction between the normal $\langle 100\rangle$ axis and the $<111>$ axis of the YSZ unit cell. The growth mechanism responsible for texture development has been studied. Based on ion channeling through grains with open crystallographic planes parallel to the assisting ion beam, Bradly et al. suggested that biaxial alignment is achieved through selective sputtering of misoriented grains that have higher sputtering yields relative to those that are aligned to the channeling direction [4]. Ressler et al. proposed texture is the result of anisotropy in ion damage, which allows selective growth of damage-tolerant planes that face the ion beam [5],[6].

Irrespective of the mechanism of nucleation, biaxially aligned YSZ grains grow out of the nucleation zone as a dendritic columnar microstructure [7]. Biaxially aligned (100) columns grow at the expense of more heavily etched misaligned columns. The anisotropy in etching rates allows the faster-growing (100) grains to shadow uniaxially aligned 
(100) columns and dominate the microstructure [8]. This growth and extinction model for biaxial alignment through shadowing and selective etching of misoriented, slowergrowing columns accounts for the in-plane alignment. The processing conditions necessary for producing highly textured films are precise, and overall film growth is slow. In addition, sputtering losses reduce the overall film thickness and, thus, longer processing times are required to produce films of equivalent thickness [9]. The sensitivity of texture development to processing variables is not well understood. For IBAD to compete with other methods of buffer-layer development, faster processing times are crucial [1].

In the present work, film development was studied primarily by evaluating the degree of texture development and the film thickness for various $\mathrm{r}$-values at a constant ion bombardment angle of $55^{\circ}$ and ion beam energy of $300 \mathrm{eV}$. A two-dimensional texture/thickness phase diagram was created from the resulting data to aid in the optimization of processing of YSZ films by IBAD.

\section{EXPERIMENTAL PROCEDURES}

The substrate was polycrystalline Hastelloy C. Strips were sheared into $1 \mathrm{~cm}^{2}$ coupons and polished with diamond paste to a final finish of $0.1 \mu \mathrm{m}$. A partially shielded $\mathrm{Si}$ control sample adjacent to the Hastelloy was used to characterize the thickness of each deposition.

The initial pressure of the deposition chamber was $1 \times 10^{-7}$ torr, which increased to $9 \times 10^{-5}$ torr with $\mathrm{Ar}$ and $\mathrm{O}_{2}$ flow into the ion source. The $\mathrm{O}_{2}$ flow rate was set at $10 \%$ of the $\mathrm{Ar}$ flow to maintain O stoichiometry in the YSZ during growth. A constant $300 \mathrm{eV}$ ion beam was generated for all depositions by setting the beam voltage to $300 \mathrm{~V}$, the discharge voltage to $40 \mathrm{~V}$, and the acceleration voltage to $100 \mathrm{~V}$. A constant bombardment angle of $55^{\circ}$ was maintained for all depositions. The current density was varied between 150 and $350 \mu \mathrm{A} / \mathrm{cm}^{2}$ by changing the beam current, and measuring with a Faraday cup the corresponding ion fluence at the substrate surface. Accurate ion fluence was ensured by proper positioning of the Faraday cup for each deposition. Electron beam evaporation was used for YSZ deposition. The deposition rate was varied between 0.64 and $3.2 \AA / \mathrm{s}$, and all depositions were carried out to a gross thickness of $1.6 \mu \mathrm{m}$, as measured by the rate monitor.

Biaxial texture was characterized with a Scintag four-circle $\mathrm{X}$-ray diffractometer and $\mathrm{Cu} \mathrm{K} \alpha$ radiation. Standard $2 \theta$ scans were used to determine the gross preferred orientation. The degree of out-of plane texture was determined by the FWHM of $\Omega$ scans of the YSZ (200) reflection by rocking through an angle of $20^{\circ}$. In-plane texture was measured by the FWHM of $\phi$ scans of the YSZ (111) reflection, and (111) pole figures were generated to qualitatively map out the degree of biaxial texture development. Profilometry was used to determine the thickness of the partially shielded Si control samples.

\section{RESULTS AND DISCUSSION}

Fig. 1 shows a series $\theta-2 \theta$ X-ray scans for films produced with increasing $r$-values. YSZ films deposited without ion assistance $(r=0)$ were essentially amorphous (the reflections at 43.5 and $50.5^{\circ}$ correspond to the Hastelloy substrate). Increasing the r-value to 1 induced a gradual increase in (111)

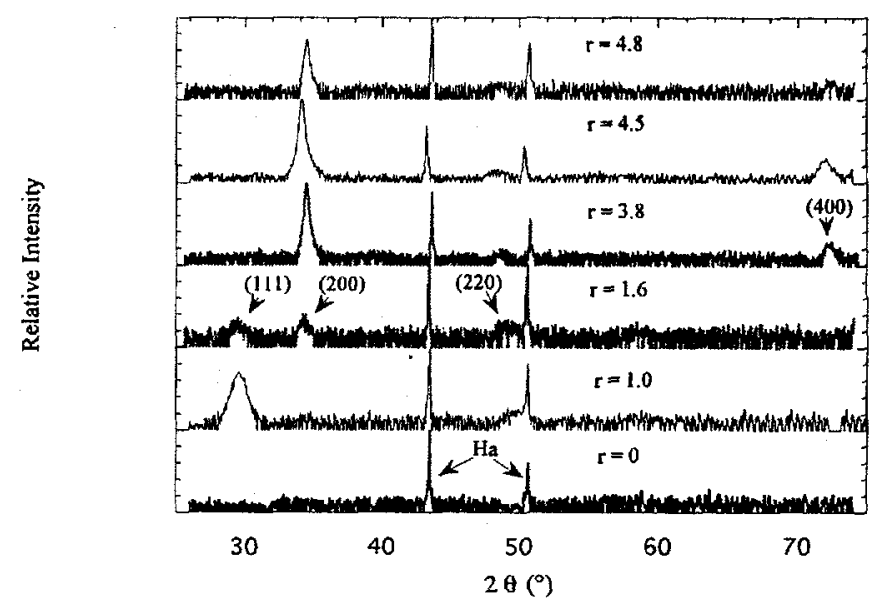

Fig. 1. $\theta-2 \theta$ scans of IBAD YSZ substrates as a function of $r$-value.

preferred growth, marked by the broad (111) peak at $30^{\circ}$, where the ions presumably provided enough surface mobility to the adatoms of YSZ to grow preferentially with a [111] direction normal to the surface. Studies of preferred growth of IBAD YSZ as a function of substrate temperature have noted formation of (111) at lower r-values and higher temperatures [10]. The ion-assisted beam limits the mobility of deposited atoms and promotes formation of a columnar microstructure that is (200) preferentially oriented. The YSZ (111) plane has the lowest surface energy and will grow parallel to the substrate surface without any ion assistance, provided that there is a small amount of substrate heating.

IBAD YSZ films produced on heated substrates show a tendency for (111) growth because of increased mobility of the adatoms [10]. Without the assisting ion beam or at low $\mathrm{r}$-values, (111) formation is more stable, whereas at larger r-values, the ion beam limits the mobility of adatoms and promotes $(200)$ growth. Films produced at an r-value of 1.6 showed both (111) and (200) grains, indicating a preferential growth change; r-values above 1.6 showed pronounced (200) preferential growth marked by an increase in relative intensity. At large r-values, the intensity dropped off because of the decreased thickness. Along with (200) and (111) orientations, there was also a small contribution of (110), which was detected by a small intensity peak at $50.1^{\circ}$. This contribution arose from the initial stages of film growth; (200) growth gradually shadowed and extinguished the growth of crystallites of other orientations, such as (110). Thus, the contribution of (110) preferential growth is generally confined to the first few atomic layers and subsides with increasing thickness [11].

Fig. 2 is a plot of relative X-ray intensity vs. r-value, which better illustrates the effect of $r$-value on preferential growth. For progressively increasing $\mathrm{r}$-values, the preferential growth changed from (111) to (200) at an $r$-value of $\approx 1.6$. Again, at large r-values, the relative intensity dropped of because of the decrease in thickness. The $(200)$ orientation dominates to a critical $r$-value $r_{c}$, at which more material is sputtered away than deposited.

The degree of c-axis, out-of-plane texture was determined by $\Omega$ scans performed on the (200) reflection. At $r$-values $>2$, all films with (200) preferred texture exhibited good c-axis alignment, as indicated by a sharp drop of the FWHM to an 


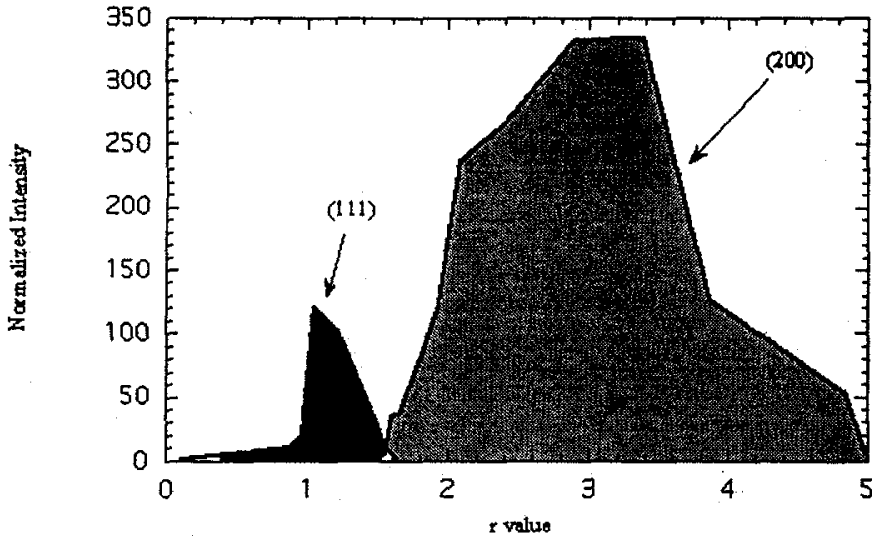

Fig. 2. $\theta-2 \theta$ relative intensity of (111) and (200) reflections vs. r-value; (200) dominates at $r>1.65$

ultimate value of $5^{\circ}$. Previous studies on various substrate materials have shown that out-of-plane texture forms readily and is mostly dependent on the surface roughness of the substrate [9]. This is expected because of growth rate anisotropy and the tendency of columnar-growth structures in YSZ, resulting in c-axes perpendicular to the surface [12].

To quantify the degree of in-plane texture, $\phi$ scans of the YSZ (111) reflection were preformed on films that exhibited good c-axis texture. The in-plane texture was studied for $r$ values of 1.6-6. Textures improved steadily with increasing $r$-values up to $\approx 3$; thereafter, the FWHM remained approximately constant for $r$-values up to $\approx 6$.

As more ions hit the surface, more of them satisfy the criteria for ion channeling and contribute to an increase in anisotropic ion etching and overgrowth of crystallites that exhibit orientations other than biaxially aligned YSZ (200) grains. Thus, increasing the ion flux allows the (200) fastergrowing grains to grow and further align themselves with respect to the ion beam. Bradly et al. [4] showed that the degree of in-plane orientation increases asymptotically to a critical value of $r\left(r_{c}\right)$, at which more material is sputtered away than deposited. Therefore, the degree of in-plane texture should also increase to a critical value. In Fig. 3, which shows this effect, the FWHM seemed to saturate at an r-value of 6 , indicating that the $8-\mathrm{cm}$ ion source is capable of achieving an FWHM of $\approx 17^{\circ}$. Glancing-angle X-ray studies have shown that the surface textures are substantially better than those of the bulk, typically $9^{\circ}$ less in the FWHM [9],[13]. This implies that an IBAD YSZ film produced with a gross in-plane texture of $19^{\circ}$ would correspond to a surface FWHM of $10^{\circ}$, and would therefore be an acceptable template for epitaxy of YBCO. The increase of in-plane alignment can be seen in Fig. 4 shows (111) pole figures for films deposited with increasing $r$-values. In all cases, the (111) pole was oriented in the direction of the bombarding ion beam.

Film thickness determined from the partially shielded Si control samples are plotted as a function of $r$-value in Fig. 5 . The decrease in thickness for increasing $r$-values follows a linear trend. From a linear fit, the $x$-intercept represents an $r_{c}$ of 6.65. Films with larger r-values have zero measurable thickness; hence, $r_{c}$ is the point at which the etching rate equals the deposition rate.

Shown in Fig. 6 is an IBAD YSZ texture/processing contour map that represents the data gathered in this study.

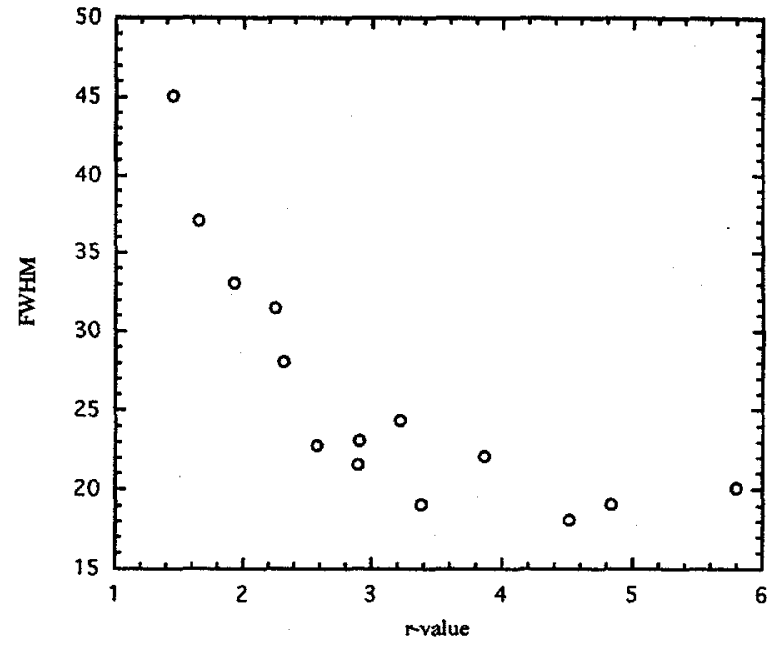

Fig. 3. In-plane texture as a function of $r$-value.

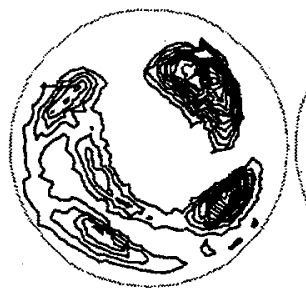

(a)

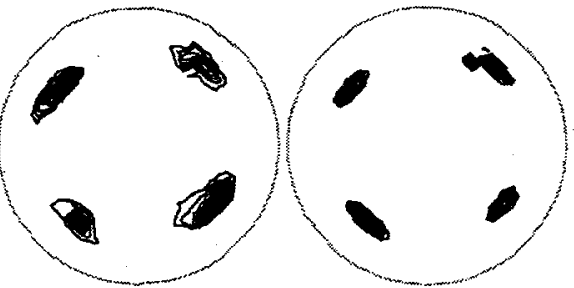

(b) (c)
Fig. 4. (111) Pole figures of IBAD YSZ films deposited with r-values of (a) 1.45 , (b) 1.94 , and (c) 3.39 .

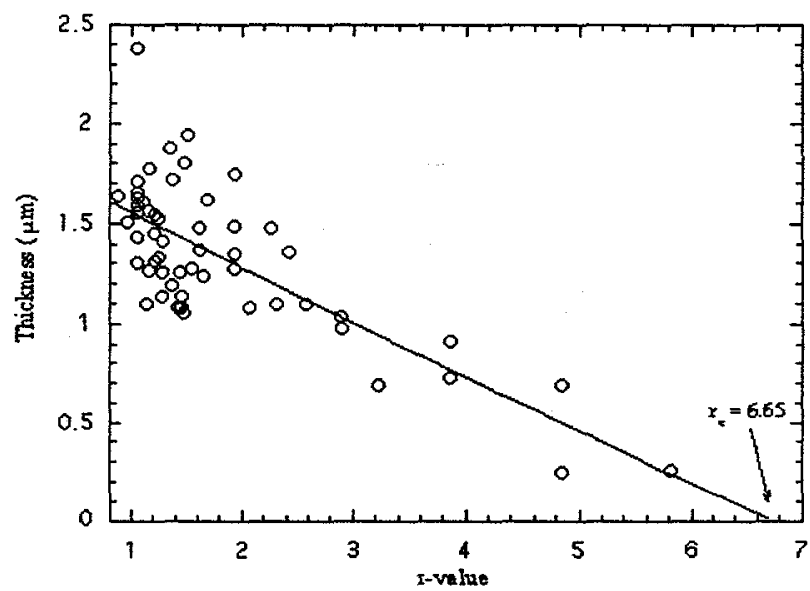

Fig. 5. Film thickness as a function of $r$-value.

Thickness and texture data were integrated to map out the zones of preferred orientation. The two most easily changed, important variables in the development of texture are the atomic and ion flux, better described as the deposition rate, and the ion current density. The data needed to generate relatively smooth contour lines (iso-FWHMs and isothicknesses) were calculated by fitting the experimental data to empirical curve fits. The data within zones that were not heavily studied were generated by extrapolating appropriate individual curves. Most of the data were gathered at deposition rates between 0.64 and $3.2 \AA / \mathrm{s}$ and at ion current densities between 150 and $350 \mu \mathrm{A} / \mathrm{cm}^{2}$, at which the tendency for biaxial texture development was the strongest. 
The zones that showed (111) and (200) preferred growth are shaded. The (200) preferred growth is presented as dark gray; it spans a large region and is present for $r$-values greater than $\approx 1.67$. The (111) zone of preferred growth is marked by lighter gray and is found between the 1 and $1.67 \mathrm{r}$-value contour boundaries. There is little overlap of the $(200)$ and (111) preferred growth that occurs at an $\mathrm{r}$-value of $\approx 1.6$ (Fig. 2). Thus, the small zone of $(111)+(200)$ can be assumed to start at the (111) transition to the (200), proceeding to extend into and overlap a very small strip of the (111) zone. Low r-values, $<1$, produced YSZ films that were for all intents and purposes amorphous. The boundary of transition from (111) to (200) preferred orientation approximately follows the 1.2 (1.67) thickness/r-value contour line. The (200) zone is present at $1.67 \leq r \leq r_{c}$.

The contour of zero thickness at the $r_{c}$ marks the boundary where the atom arrival rate and etching rate reach unity, and preferred (200) film growth begins. However, this boundary is not as defined as the line suggests. The processing zone between the zero and 0.2 thickness contour boundaries was very susceptible to ion bombardment. Such large r-values introduced increased amounts of ion damage and stress between the film and substrate and was evident by visual inspection that revealed poor cohesion and delamination of the films deposited within this zone.

The region marked with heavy lines within the (200) zone represents biaxial texture development. Contained in this area are iso-FWHMs (lines of equal biaxial texture). FWHM data were obtained from films produced with ion current densities of $150-350 \mu \mathrm{A} / \mathrm{cm}^{2}$. No FWHM data were obtained for very low deposition rates, which require excessively long processing times to reach the $1.6 \mu \mathrm{m}$ thickness criterion. Thus, the dashed borders represent the cutoff of data analysis. However, the biaxial texture zone can be expected to extend past the dashed regions following the isolines, eventually being pinched off at an $r$-value of $\approx 3$, which corresponds to both low and high deposition-rate and current-density coordinates. This zone can be expected to extend through the (200) zone, terminating at the $r_{c}$ contour boundary.

\section{CONCLUSIONS}

An IBAD processing map was generated to tailor the degree of YSZ in-plane texture and film thickness as a function of ion-to-atom arrival flux ratio. Films produced at $\mathbf{r}-$ values $<1.67$ displayed (111) texture, becoming increasingly amorphous with decreasing r-value. Films at $r$-values $>1.67$ exhibited (200) biaxial texture, approaching a FWHM of $17^{\circ}$ at the critical r-value of 6.65 .

\section{REFERENCES}

[1] Finnemore, D. K., Gray, K. E., Maley, M. P., Welch, D. O., Christen, D. K., and Kroeger, D. M., "Coated conductor development: an assessment," Physica C, vol. 320, pp. 1-8, 1999.

[2] Sheel, H. J., Berkowski, M., and Chabot, B., "Problems in epitaxial growth of high- $T_{\mathrm{c}}$ superconductors," J. Crys. Growth, vol. 115, pp. 1930,1991

[3] Iijima, Y., and Matsumoto, K., "High-temperature-superconductor coated conductors: technical progress in Japan," Supercond. Sci. Technol., vol. 13, pp. 68-81, 2000.

[4] Bradley, R., Harper, J., and Smith, D., "Theory of thin-film orientation by ion bombardment during deposition," $J$. Appl. Phys., vol. 60, pp. 4160-4164, 1996.

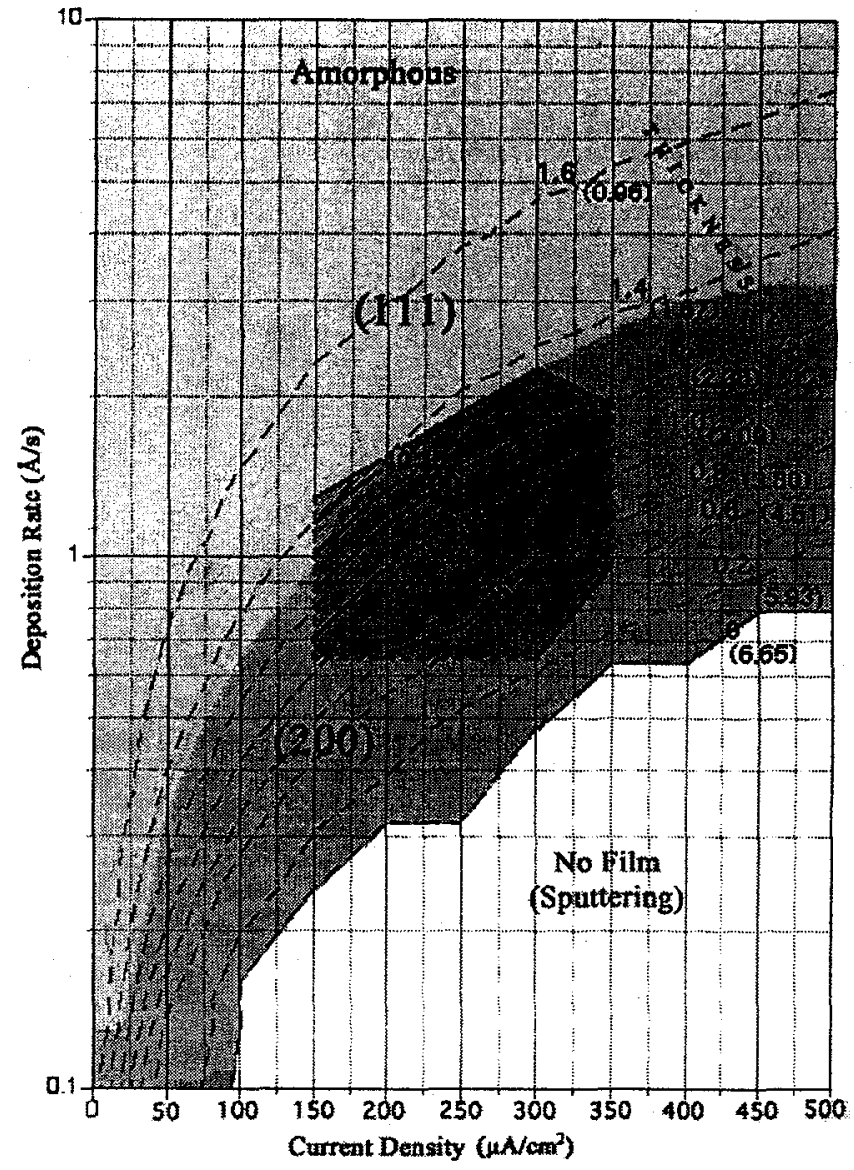

Fig. 6. Orientation of YSZ as a function of IBAD processing conditions.

[5] Ressler, K. G., Sonnenberg, N., and Cima, M. J., "Mechanism of biaxial alignment of oxide thin films during ion-beam-assisted deposition," J. Am. Ceram. Soc., vol. 80, pp. 2637-2648, 1997.

[6] Ressler, K. G., Sonnenberg, N., and Cima, M. J., "The relation between ion damage anisotropy and IBAD YSZ biaxial alignment," IEEE Trans. Appl. Supercond, vol. 7, pp. 1432-1435, 1997.

[7] Holzapfel, B., Betz, V., Schlafer, D., Bauer, H. D., and Schultz, L., "Microstructure and texture evolution in oxide films prepared by ionbeam assisted deposition," IEEE Trans. Appl. Supercond., vol. 9, pp. 1479-1482, 1999.

[8] McIntyre, P. C., Ressler, K. G., Sonnenberg, N., and Cima, M. J., "Transmission electron microscopy investigation of biaxial alignment development in YSZ films fabricated using ion beam assisted deposition," J. Vac. Sci. Technol., vol. 14, No. 1, pp. 1232-1244, 1996.

[9] Chudzik, M. P., Erck, R., and Lanagan, M. T., "Processing dependence of biaxial texture in yttria-stabilized zirconia by ionbeam-assisted deposition," IEEE Trans. Appl. Supercond., vol. 9, pp. 1490-1493, 1999

[10] Mao, Y. J., Ren, C. X., Yuan, J., Zhang, F., Liu, X. H., and Zou, S. C., "Study on the growth of biaxially aligned yttria-stabilized zirconia films during ion beam assisted deposition," J. Vac. Sci. Technol., vol. 15, pp. $331-342,1997$.

[11] Weismann, J., Dzick, J., Hoffman, J., Heinemann, K., and Freyhardt, H. C., "Growth mechanisms of biaxially textured YSZ films deposited by ion-beam-assisted deposition," J. Mater. Res., vol. 13, pp. 31493152,1998

[12] Norton, D. P., Lowndes, D. H., Budai, J. D., and Christen, D. K., "High critical current densities in $\mathrm{YBa}_{2} \mathrm{Cu}_{3} \mathrm{O}_{7 \times x}$ films on polycrystalline zirconia," Appl. Phys. Lett., vol. 57, pp. 1164-1166, 1990.

[13] Knierim, A., Auer, R., Geerk, J., Li, Y., Linker, G., Meyer, O., Reiner, J., Schweiss, P., and Smithey, R., "Influence of the process parameters on the growth of YSZ layers prepared by ion-beamassisted deposition (IBAD)," Nucl. Instrum. Methods, vol. B, No. 127/128, pp. 832-836, 1997. 